\title{
Nonlinear electrostatic modes in astrophysical plasmas with charged dust distributions
}

\author{
F. Verheest ${ }^{1,2}$ and V. V. Yaroshenko ${ }^{3}$ \\ 1 Sterrenkundig Observatorium, Universiteit Gent, Krijgslaan 281, 9000 Gent, Belgium \\ e-mail: Frank.Verheest@uGent.be \\ 2 School of Physics, University of KwaZulu-Natal, Private Bag X54001, Durban 4000, South Africa \\ 3 Max-Planck-Institut für extraterrestrische Physik, 85741 Garching, Germany
}

Received 27 March 2009 / Accepted 2 June 2009

ABSTRACT

\begin{abstract}
Context. Most theoretical investigations of wave phenomena in dusty plasmas have treated charged dust as additional heavy negative ions in standard multispecies models. Many dusty plasma experiments use monodisperse grains, but charged (and neutral) grains in space environments come in a whole range of sizes and compositions, hence in masses and charges. Dust density distributions having a power-law decay with size are often observed in planetary rings.

Aims. There is a need to investigate larger solitary structures, since wave descriptions involving charged dust distributions have usually been limited to linear or weakly nonlinear modes.

Methods. Sagdeev pseudopotential methods describe large-amplitude solitary waves in a co-moving reference frame and are adapted to include dust distributions, using mass and charge, or alternatively size, as additional variables. Observed power-law distributions lead to descriptions involving hypergeometric functions.

Results. Lower limits for possible solitary wave velocities follow from requiring that the Sagdeev pseudopotentials have a local maximum for the undisturbed conditions, whereas upper limits come from ensuring that dust densities remain real. Nonlinear amplitudes increase with solitary wave velocities and relative electron temperature. Under similar plasma conditions, dust size distributions admit slower solitary wave solutions, with correspondingly smaller amplitudes than given by monodisperse dust models. For typical planetary ring conditions, the difference can be quite appreciable.
\end{abstract}

Key words. plasmas - waves - planets: rings

\section{Introduction}

This paper combines two strands of current research in the description of space and astrophysical plasmas. On the one hand, there is the continuing modeling of exact large scale nonlinear solitary waves for many different plasma compositions, on which a vast literature has sprung up since the pioneering work by Sagdeev (1966). Only some immediately relevant references will be given below, there being no hope of doing this extended domain any semblance of justice. Solitary waves seen in space have usually been in the form of bipolar pulses in the electric field or humps and dips in the electrostatic potential and observed in many parts of the magnetosphere: for instance, the auroral region (Bounds et al. 1999), the bow shock (Bale et al. 1998), the magnetosheath (Pickett et al. 2003), and the plasma sheet boundary region (Matsumoto et al. 1994; Frantz et al. 1998), to quote just a selected few.

On the other hand, the theoretical treatment of wave phenomena in dusty plasmas (see, e.g., monographs by Bliokh et al. 1995; Verheest 2000; Shukla \& Mamun 2002) has mostly introduced charged dust as heavy negative ion species, in models that are extensions of earlier work on more usual plasmas. While many dusty plasma experiments have used manufactured monodisperse grains, charged (and neutral) grains in space and astrophysical situations come in a whole range of sizes and compositions, hence also masses and charges. Moreover, in situ observations of planetary environments by space missions have often shown that dust distributions are characterized by power-law decays of dust densities with size (Gurnett et al. 1983; Showalter et al. 1992; Showalter \& Cuzzi 1993; Kempf et al. 2006; Kurth et al. 2006; Kempf et al. 2008; Yaroshenko et al. 2009).

Incorporating the effect of charged dust distributions in the description of waves in dusty plasmas has mostly been restricted to linear or weakly nonlinear modes (Tripathi \& Sharma 1996; Brattli et al. 1997; Meuris et al. 1997; de Juli \& Schneider 1998; Yaroshenko et al. 2001), so we describe large-amplitude, nonlinear solitary waves in space plasmas that contain charged dust distributions, to which end we have to adapt the Sagdeev pseudopotential formalism.

The plan of the paper is as follows. After this introduction, Sect. 2 shows how dust distributions within a range of charges and masses can be dealt with in a Sagdeev pseudopotential formalism, which is specialized in Sect. 3 to grain size distributions with a power-law decay, as often observed in space plasmas. Weakly nonlinear solitary structures is looked at in Sect. 4, whereas Sect. 5 treats the fully nonlinear case and delimits the existence ranges in compositional parameter space in terms of physical concepts. A comparison is then made with monodisperse dust descriptions in Sect. 6, and Sect. 7 sums up our conclusions.

\section{Nonlinear formalism}

First we very briefly repeat some of the steps needed to describe complex plasmas with polydisperse charged dust, with emphasis on a methodologically correct way. We distinguish between the electrons and ions treated by standard theory, on the one 
hand, and the dust distributions, described by appropriate integrals over the charge and mass distributions, on the other.

For electrostatic problems, the waves are longitudinal, so that only scalar velocities are needed. To avoid all ambiguities, we start from a microscopic distribution function $f_{\mathrm{d}}(x, w, t, q, m)$ for the dust in phase space, which incorporates the dust charge $q$ and the mass $m$ as continuous, independent microscopic phase space variables, besides $x, t$, and the phase space parallel velocity $w$. This is governed by an extended Vlasov-type kinetic equation (Varma 2000; Verheest et al. 2002a, 2003; Verheest \& Cattaert $2004 a, b)$, which for electrostatic modes reads as

$\frac{\partial f_{\mathrm{d}}}{\partial t}+w \frac{\partial f_{\mathrm{d}}}{\partial x}-\frac{q}{m} \frac{\partial \varphi}{\partial x} \frac{\partial f_{\mathrm{d}}}{\partial w}=0$,

where the microscopic laws of motion $\dot{x}=w$ and $\dot{w}=$ $-(q / m)(\partial \varphi / \partial x)$ were used, and $\varphi$ is the electrostatic potential. Integrations over transverse directions were carried out and for dust grains with constant charges and masses there are no $\partial\left(\dot{q} f_{\mathrm{d}}\right) / \partial q$ and $\partial\left(\dot{m} f_{\mathrm{d}}\right) / \partial m$ contributions, even though $q$ and $m$ cover a whole range.

After multiplication with some power of the microscopic velocities, integration of (1) over all $q, m$ and $w$ yields the traditional chain of moment equations in physical space for one-dimensional motion. As explained elsewhere (Verheest \& Cattaert 2003), different charge and mass weightings compel one to introduce a whole sequence of velocities and higher order moments, where the same order in $w$ is combined with different powers of $q$ and $m$. To avoid these complications, we postpone the integration over the new phase space variables $q$ and $m$ and restrict ourselves first to integrations solely over the microscopic velocity $w$.

More details about this half-way house procedure are given elsewhere (Verheest \& Cattaert 2004a,b), and, in this sense, we define the zeroth velocity moment of $f_{\mathrm{d}}$ as

$\widetilde{n}_{\mathrm{d}}=\int f_{\mathrm{d}} \mathrm{d} w$

such that the real physical dust density obtains as

$n_{\mathrm{d}}=\int \widetilde{n}_{\mathrm{d}} \mathrm{d} q \mathrm{~d} m=\int f_{\mathrm{d}} \mathrm{d} w \mathrm{~d} q \mathrm{~d} m$.

In this intermediate description, variables will be indicated with a tilde, to show that these still contain variable $q$ and $m$, integrations over which have to be carried out at a later stage. As $f_{\mathrm{d}}$ is normalized to give $n_{\mathrm{d}}$, its moments of order one are defined as

$\widetilde{v}_{\mathrm{d}}=\frac{1}{\widetilde{n}_{\mathrm{d}}} \int f_{\mathrm{d}} w \mathrm{~d} w$

such that the dust fluid velocity is given by

$v_{\mathrm{d}}=\frac{1}{n_{\mathrm{d}}} \int \widetilde{n}_{\mathrm{d}} \widetilde{v}_{\mathrm{d}} \mathrm{d} q \mathrm{~d} m=\frac{1}{n_{\mathrm{d}}} \int f_{\mathrm{d}} w \mathrm{~d} w \mathrm{~d} q \mathrm{~d} m$.

Hence, the first moment equations for the dust are similar to the continuity and momentum equations for ordinary plasma species, but with a different interpretation, because $q$ and $m$ are variable. We start from

$\frac{\partial \widetilde{n}_{\mathrm{d}}}{\partial t}+\frac{\partial}{\partial x}\left(\widetilde{n}_{\mathrm{d}} \widetilde{v}_{\mathrm{d}}\right)=0$

$\left(\frac{\partial}{\partial t}+\widetilde{v}_{\mathrm{d}} \frac{\partial}{\partial x}\right) \widetilde{v}_{\mathrm{d}}=-\frac{q}{m} \frac{\partial \varphi}{\partial x}$

written for cold dust, without pressure effects.
We use the stationary form $(\partial / \partial t=0)$ of the equation of continuity (6) and motion (7), to get first integrals expressing mass flux and momentum conservation, as it is easier to work in the wave frame moving with the nonlinear solitary structure. This change in reference frame entails a redefinition of the microscopic distribution functions $f_{\mathrm{d}}$ in terms of the microscopic velocities.

Since in an inertial frame all species are macroscopically at rest in the absence of solitary structures, this holds for the cold dust for all $q$ and $m$ within the admissible ranges. In the wave frame (6) expresses mass conservation as

$\widetilde{n}_{\mathrm{d}} \widetilde{v}_{\mathrm{d}}=\widetilde{n}_{\mathrm{d} 0} V$

where $V$ is the velocity of the solitary wave as it would be seen in an inertial frame, but, in the wave frame, $V$ corresponds to the undisturbed plasma velocities far away from the solitary wave. Hence we have also

$n_{\mathrm{d}} v_{\mathrm{d}}=\int \widetilde{n}_{\mathrm{d}} \widetilde{v}_{\mathrm{d}} \mathrm{d} q \mathrm{~d} m=V \int \widetilde{n}_{\mathrm{d} 0} \mathrm{~d} q \mathrm{~d} m=n_{\mathrm{d} 0} V$.

Eliminating $\widetilde{v}_{\mathrm{d}}$ then between the stationary forms of (6) and (7) yields in the standard way that

$\widetilde{n}_{\mathrm{d}}=\frac{\widetilde{n}_{\mathrm{d} 0}}{\sqrt{1-\frac{2 q \varphi}{m V^{2}}}}$

The plasma electrons and ions will be described by the usual Boltzmann distributions,

$n_{\mathrm{e}}=n_{\mathrm{e} 0} \exp \left[\frac{e \varphi}{T_{\mathrm{e}}}\right]$

$n_{\mathrm{i}}=n_{\mathrm{i} 0} \exp \left[-\frac{e \varphi}{T_{\mathrm{i}}}\right]$

with respective kinetic temperatures $T_{\mathrm{e}}$ and $T_{\mathrm{i}}$. In the undisturbed conditions charge neutrality demands that

$n_{\mathrm{i} 0} e-n_{\mathrm{e} 0} e+\int \widetilde{n}_{\mathrm{d} 0} q \mathrm{~d} q \mathrm{~d} m=0$.

The basic description is closed by Poisson's equation,

$\varepsilon_{0} \frac{\mathrm{d}^{2} \varphi}{\mathrm{d} x^{2}}+n_{\mathrm{i}} e-n_{\mathrm{e}} e+\int \widetilde{n}_{\mathrm{d}} q \mathrm{~d} q \mathrm{~d} m=0$.

After multiplication by $\mathrm{d} \varphi / \mathrm{d} x$ and integration with respect to $x$ we get

$\frac{1}{2}\left(\frac{\mathrm{d} \varphi}{\mathrm{d} x}\right)^{2}+S(\varphi)=0$,

where the Sagdeev pseudopotential $S(\varphi)$ is given by

$$
\begin{aligned}
S(\varphi)= & \frac{n_{\mathrm{e} 0} T_{\mathrm{e}}}{\varepsilon_{0}}\left(1-\exp \left[\frac{e \varphi}{T_{\mathrm{e}}}\right]\right) \\
& +\frac{n_{\mathrm{i} 0} T_{\mathrm{i}}}{\varepsilon_{0}}\left(1-\exp \left[-\frac{e \varphi}{T_{\mathrm{i}}}\right]\right) \\
& +\frac{V^{2}}{\varepsilon_{0}} \int \widetilde{n}_{\mathrm{d} 0} m\left\{1-\sqrt{1-\frac{2 q \varphi}{m V^{2}}}\right\} \mathrm{d} q \mathrm{~d} m .
\end{aligned}
$$

Now (14) has the form of an energy integral in classical mechanics, for particles with unit mass in a conservative force field, 
$\varphi$ playing the role of a coordinate and $x$ that of time. The discussion can thus be given with the mechanical analogy in mind. Examples of $S(\varphi)$ will be illustrated in Figs. 4 and 5, but these can only be generated once we have determined for which compositional plasma parameters and solitary wave velocities nonlinear profiles can exist.

It is seen that $S(0)$ and $(\mathrm{d} S / \mathrm{d} \varphi)(0)=0$, and in order to have the possibility of solitary structures, we need the proper convexity in the origin, namely that

$\frac{\mathrm{d}^{2} S}{\mathrm{~d} \varphi^{2}}(0)=\frac{1}{V^{2}} \int \frac{\widetilde{n}_{\mathrm{d} 0} q^{2}}{\varepsilon_{0} m} \mathrm{~d} q \mathrm{~d} m-\frac{n_{\mathrm{e} 0} e^{2}}{\varepsilon_{0} T_{\mathrm{e}}}-\frac{n_{\mathrm{i} 0} e^{2}}{\varepsilon_{0} T_{\mathrm{i}}}<0$,

ensuring that the origin is a local maximum for $S(\varphi)$, viz. that $S(\varphi)<0$ for $\varphi \neq 0$ but in the immediate vicinity of $\varphi=0$. Introducing a global Debye length $\lambda_{\mathrm{D}}$, through

$\frac{1}{\lambda_{\mathrm{D}}^{2}}=\frac{1}{\lambda_{\mathrm{De}}^{2}}+\frac{1}{\lambda_{\mathrm{Di}}^{2}}=\frac{n_{\mathrm{e} 0} e^{2}}{\varepsilon_{0} T_{\mathrm{e}}}+\frac{n_{\mathrm{i} 0} e^{2}}{\varepsilon_{0} T_{\mathrm{i}}}$,

as well as the correct definition of the total dust plasma frequency $\omega_{\text {pd }}$, through

$\omega_{\mathrm{pd}}^{2}=\int \frac{\widetilde{n}_{\mathrm{d} 0} q^{2}}{\varepsilon_{0} m} \mathrm{~d} q \mathrm{~d} m$,

allows us to rewrite the so-called soliton condition (16) as

$V_{\mathrm{da}}:=\omega_{\mathrm{pd}} \lambda_{\mathrm{D}}<V$

This requires possible solitary structures to be superacoustic with respect to the dust-acoustic velocity $V_{\mathrm{da}}$.

Note that while this is a necessary condition, it is not sufficient to guarantee that solitary modes will exist. There are further restrictions on $V$ that follow from the occurrence (or not) of roots for $S(\varphi)$ outside $\varphi=0$.

\section{Dust size distributions}

For astrophysical plasmas, the main difficulties lie not so much in estimating $T_{\mathrm{e}}$ and $T_{\mathrm{i}}$, but to know how the dust distributions $\widetilde{n}_{\mathrm{d} 0}$ vary with $q$ and $m$, and in what ranges. To simplify the general description and have a manageable discussion when dealing with planetary dust rings, we will assume in this section that we can use the size $a$ of the dust grains as the variable factor.

Now we assume that the dust grains all have equilibrium charges determined from the orbital-motion-limited (OML) or spherical probe charging theory (see, e.g., Bliokh et al. 1995; Verheest 2000; Shukla \& Mamun 2002), where the dust is charged by electron and ion currents to the grains. For space dusty plasmas, which usually are weakly collisional, the OML theory represents a good approximation. Measurements of the particle charges made by the Cassini orbiter within Saturn's E-ring, e.g., are in quite reasonable agreement when compared to theoretical predictions based on the OML theory (Kempf et al. 2006).

In this model the dust charge is negative and proportional to the grain size $a$, in a given plasma environment. Furthermore, for grains composed of similar material the mass varies with $a^{3}$. Thus we can put $q(a)=-Q a$ and $m(a)=M a^{3}$, so that we are actually dealing with a dust size distribution and the (redefined) distribution function $f_{\mathrm{d}}(x, w, t, a)$ will now depend on $a$ as the parameter characterizing the dust distribution, instead of on $q$ and $m$ separately.
Next we take a standard power-law distribution, i.e. we assume that

$\widetilde{n}_{\mathrm{d} 0}(a)=\int f_{\mathrm{d} 0}(w, a) \mathrm{d} w=K a^{-\beta}$

in an interval $\left[a_{\min }, a_{\max }\right]$. More complicated size distributions combine an exponential behaviour with a power-law decay (Raadu 2001) and lead to a description in terms of $\kappa$-distributions (Summers \& Thorne 1991; Mace \& Hellberg 1995; Hellberg et al. 2005).

Power-law density decreases with size, as in (20), are typical for different space plasma environments. Distributions of this sort have been observed in planetary ring plasmas, with powerlaw indices $\beta=4.6$ for the F ring of Saturn (Showalter et al. 1992) and $\beta=6$ (Showalter \& Cuzzi 1993), or $\beta=7$ (Gurnett et al. 1983) for the $\mathrm{G}$ ring. The Voyager observations that have yielded these power-law indices and related information about planetary dust in the rings of the Jovian planets are more than two decades old. However exciting these observations may have been, they essentially correspond to snapshots from the flybys of the Voyager spacecraft on their Grand Tour of the solar system.

Hence the anticipation to more extended and precise data from the Cassini mission, which from the middle of 2004 onwards have reached us and which now confirm that dust size distributions are indeed well described by power laws. During most encounters with Saturn's rings in the vicinity of the icy moon Enceladus, the power law index inferred from dust impact measurements was found to be between $4.8<\beta<5.4$ (Kempf et al. 2008), while the Cassini Radio and Plasma Wave Science instrument predicted $\beta \simeq 6.4 \pm 1.0$ (Kurth et al. 2006). Estimates based on Langmuir probe plasma measurements yielded even steeper dust distributions with $\beta \simeq 7-8$ (Yaroshenko et al. 2009).

Moreover, for typical solar system dust size distributions the parameter $a_{\min } / a_{\max }$ is small enough to simplify some of the subsequent expressions for $\beta$ values between 4 and 7 . Powerlaw distributions are fairly generic for other astrophysical plasmas, as in molecular clouds, based on considerations that smaller grains are more numerous than larger ones, although detailed observations outside the solar system are lacking for the time being.

We find for the undisturbed dust charge density that

$$
\begin{aligned}
\sigma_{\mathrm{d} 0} & =\int_{a_{\min }}^{a_{\max }} \widetilde{n}_{\mathrm{d} 0} q \mathrm{~d} a=-\frac{K Q}{\beta-2}\left(\frac{1}{a_{\min }^{\beta-2}}-\frac{1}{a_{\max }^{\beta-2}}\right) \\
& \simeq-\frac{K Q}{(\beta-2) a_{\text {min }}^{\beta-2}}<0 .
\end{aligned}
$$

In accordance with observational results, we often will in this and the following expressions assume that $\beta>4$. The last expression of $\sigma_{\mathrm{d} 0}$ is a valid approximation when $a_{\min }$ is much smaller than $a_{\max }$, in which case $\sigma_{\mathrm{d} 0}$ is weighted towards the more numerous but smaller dust grains. The latter are unfortunately difficult to ascertain observationally, since they could be well below the sensitivity of the detectors.

In the same vein, applying (20) to (18), we note that

$$
\omega_{\mathrm{pd}}^{2}=\frac{K Q^{2}}{\varepsilon_{0} M \beta}\left(\frac{1}{a_{\min }^{\beta}}-\frac{1}{a_{\max }^{\beta}}\right) \simeq \frac{K Q^{2}}{\varepsilon_{0} M \beta a_{\min }^{\beta}} .
$$

This is needed, e.g., for a discussion of linear dust-acoustic waves (Rao et al. 1990; Meuris 1997). 
Combining now (15) and (20) gives

$$
\begin{aligned}
S(\varphi)= & \frac{n_{\mathrm{e} 0} T_{\mathrm{e}}}{\varepsilon_{0}}\left(1-\exp \left[\frac{e \varphi}{T_{\mathrm{e}}}\right]\right) \\
& +\frac{n_{\mathrm{i} 0} T_{\mathrm{i}}}{\varepsilon_{0}}\left(1-\exp \left[-\frac{e \varphi}{T_{\mathrm{i}}}\right]\right) \\
& +\frac{V^{2} K M}{\varepsilon_{0}} \int_{a_{\min }}^{a_{\max }} a^{3-\beta}\left\{1-\sqrt{1+\frac{2 Q \varphi}{M a^{2} V^{2}}}\right\} \mathrm{d} a .
\end{aligned}
$$

We start by discussing the behaviour of $S(\varphi)$ for $\varphi>0$ and note that it is mathematically well defined in the whole of that range. Moreover, $S(\varphi) \rightarrow-\infty$ for $\varphi \rightarrow+\infty$, as ultimately the electron term dominates. At large positive $\varphi$ the dust contribution goes as $-\sqrt{\varphi}$, multiplied by positive constants and by the integral of $a^{2-\beta}$ over $a$ in the range $\left[a_{\min }, a_{\max }\right]$. This integral results in

$$
\frac{a_{\max }^{3-\beta}-a_{\min }^{3-\beta}}{3-\beta} \text {. }
$$

and such expressions are always positive and finite, regardless of the exponent, here $3-\beta$, but others will be encountered. When $a_{\min } \ll a_{\max }$, it is the small dust grains that dominate for $\beta>3$, whereas for $\beta<3$ the large grains prevail. For $\beta=3$, we have $\ln \left(a_{\max } / a_{\min }\right)$. This holds, mutatis mutandis, for other values than 3 , even noninteger ones.

The required convexity (16) of $S(\varphi)$ in $\varphi=0$ ensures that $S(\varphi)<0$ for $\varphi>0$ but small. Combined with $S(\varphi) \rightarrow-\infty$ for $\varphi \rightarrow+\infty$ this means that positive roots for $S(\varphi)$, if they exist at all, must come in pairs, counting possible double roots as two. From the generic behaviour of Sagdeev pseudopotentials we know that double layers may represent limiting values for a region in parameter space in which solitary waves may occur (Baboolal et al. 1990). A double layer is characterized by the coincidence of a zero of the Sagdeev pseudopotential with a zero of its derivative, i.e., with a charge neutral point. In a double layer the electrostatic potential varies from one value to another.

If such double layers can occur, the corresponding structure velocity $V=V_{\mathrm{dl}}$, for given compositional parameters, represents the upper limit on $V$ (Cattaert et al. 2005; Verheest \& Pillay 2008; Verheest et al. 2008). At the same time, a necessary condition for the existence of solitary structures is that they be superacoustic, so that taken together the range in structure velocities is $V_{\mathrm{da}}<V \leqslant V_{\mathrm{dl}}$. We know from countless discussions of Sagdeev pseudopotential applications that the solitary wave amplitudes increase with increasing $V$, so that in the case of double layer limitations, the latter amplitude is indeed the maximal amplitude possible in that range.

Because $S(\varphi)$ is mathematically well defined for all $\varphi>0$, we can conclude that, if the compositional parameters permit the occurrence of positive double layers, positive solitary waves will exist for all $V_{\mathrm{da}}<V<V_{\mathrm{dl}}$. Provided that on a certain polarity side (here $\varphi>0$ ) there are no physical restrictions on $V_{\mathrm{da}}<V$ other than the occurrence of double layers, solitary waves of small amplitudes will be encountered for $V$ marginally larger than $V_{\mathrm{da}}$, even if the limiting double layers themselves are of finite amplitude. This can be checked on the properties of solitary waves in plasma models which are far simpler to discuss than the model under consideration here (Cattaert et al. 2005; Verheest \& Pillay 2008; Verheest et al. 2008).

So in this case, where the only restrictions on $\varphi>0$ come in the form of (possibly large) double layers, we conclude that if there are large positive solitary waves for $V \lesssim V_{\mathrm{dl}}$, there should also exist arbitrarily small ones, of the same polarity, for $V$ just larger than $V_{\mathrm{da}}$. Then a weakly nonlinear approach, based on an expansion in $\varphi$ up to lowest nontrivial order, as used in the next section, gives an indication of whether solitary waves of the required polarity can exist.

Unfortunately, great care has to be taken in using this type of argument, because for certain parameter regimes and solitary wave velocities, or in the presence of other physical limitations like infinite compression or total rarefaction of certain plasma species, there might exist large solitary waves having no weak amplitude counterparts. Running ahead of ourselves, we will see in the next section that there can be no weak solitary waves having a positive polarity. In that case, also larger amplitude positive solitary waves or double layers are not possible, and we can restrict all attention to structures having a negative polarity.

On the negative $\varphi$ side, the picture is completely different, as the integrand is no longer real when $1+2 Q \varphi / M a^{2} V^{2}<0$, for $a_{\min } \leqslant a \leqslant a_{\max }$. This implies a lower limit,

$\varphi_{\mathrm{c}}=-\frac{M a_{\min }^{2} V^{2}}{2 Q}$

below which the integration over the dust contribution loses its meaning, and thus $\varphi$ is on the negative potential side limited by $\varphi_{\mathrm{c}} \leqslant \varphi<0$.

\section{Weakly nonlinear solitary waves}

In the light of the foregoing discussion, we will first investigate the weakly nonlinear solitary waves, for which we expand $S(\varphi)$ in powers of $\varphi$ up to $\varphi^{3}$. This gives

$S(\varphi) \simeq \frac{A}{2} \varphi^{2}+\frac{B}{6} \varphi^{3}$,

where the coefficients $A$ and $B$ are given as

$$
\begin{aligned}
A= & \frac{\omega_{\mathrm{pd}}^{2}}{V^{2}}-\frac{1}{\lambda_{\mathrm{D}}^{2}} \\
B= & \frac{\varepsilon_{0}}{e n_{\mathrm{i} 0} \lambda_{\mathrm{Di}}^{4}}-\frac{\varepsilon_{0}}{e n_{\mathrm{e} 0} \lambda_{\mathrm{De}}^{4}} \\
& -\frac{3 K Q^{3}}{\varepsilon_{0} M^{2} V^{4}(\beta+2)}\left(\frac{1}{a_{\mathrm{min}}^{\beta+2}}-\frac{1}{a_{\mathrm{max}}^{\beta+2}}\right)
\end{aligned}
$$

We see that $A$ is annulled for $V=V_{\mathrm{da}}$, defined in (19). In order to obtain a nonlinear solution of (26), both the quadratic and cubic terms in the expansion must contribute equally, and hence we take $V=V_{\mathrm{da}}(1+\delta)$, with $\delta$ small, so that $V_{\mathrm{da}} \delta$ is of order $\varphi$. To first order in $\delta$, we have $A=-2 \delta / \lambda_{\mathrm{D}}^{2}$, whereas in $B$ we just replace $V$ by $V_{\mathrm{da}}$, the correction in $\delta$ there contributing to higher order terms. This yields

$$
\begin{aligned}
B & \simeq \frac{3 \varepsilon_{0} \beta^{2}}{\left(\beta^{2}-4\right) \sigma_{\mathrm{d} 0} \lambda_{\mathrm{D}}^{4}}-\frac{\varepsilon_{0}}{e n_{\mathrm{e} 0} \lambda_{\mathrm{De}}^{4}}+\frac{\varepsilon_{0}}{e n_{\mathrm{i} 0} \lambda_{\mathrm{Di}}^{4}} \\
& =\frac{\varepsilon_{0}}{\sigma_{\mathrm{d} 0}}\left[\frac{2 \beta^{2}+4}{\left(\beta^{2}-4\right) \lambda_{\mathrm{D}}^{4}}+\frac{1}{n_{\mathrm{e} 0} n_{\mathrm{i} 0}}\left(\frac{n_{\mathrm{e} 0}}{\lambda_{\mathrm{Di}}^{2}}+\frac{n_{\mathrm{i} 0}}{\lambda_{\mathrm{De}}^{2}}\right)^{2}\right],
\end{aligned}
$$

again up to small terms in powers of $a_{\min } / a_{\max }$. For negative dust we find that $\sigma_{\mathrm{d} 0}<0$ and hence $B<0$, which indicates that only negative solitary waves exist, always under the assumption that $\beta>4$. If we compare the expression for $B$ with that for monodisperse dust (Verheest et al. 2002b),

$$
B=\frac{\varepsilon_{0}}{\sigma_{\mathrm{d} 0}}\left[\frac{2}{\lambda_{\mathrm{D}}^{4}}+\frac{1}{n_{\mathrm{e} 0} n_{\mathrm{i} 0}}\left(\frac{n_{\mathrm{e} 0}}{\lambda_{\mathrm{Di}}^{2}}+\frac{n_{\mathrm{i} 0}}{\lambda_{\mathrm{De}}^{2}}\right)^{2}\right],
$$


we see that the influence of the dust size distribution is to increase $|B|$, sometimes considerably, since

$\frac{2 \beta^{2}+4}{\beta^{2}-4}=2+\frac{12}{\beta^{2}-4}$.

Not really surprisingly, however, this effect diminishes at increasing $\beta$ : with a very steep fall-off in the dust distribution with size, the smallest grains becomes paramount, and this is then very close to a monodisperse situation.

Turning now to the standard one-soliton solution of (26), we have that

$\varphi=-\frac{6 \delta}{|B| \lambda_{\mathrm{D}}^{2}} \operatorname{sech}^{2}\left(\frac{1}{\lambda_{\mathrm{D}}} \sqrt{\frac{\delta}{2}} x\right)$,

which exhibits typical solitary hump or dip profiles, in the present case, potential dips. As is well known, $\delta>0$ means that the solitons are superacoustic with respect to $V_{\mathrm{da}}$, the linear phase velocity in the long-wavelength limit. Moreover, $\delta$ also determines the soliton amplitude and its inverse width, resulting in the usual saying that "taller is faster and slimmer". An increase in $|B|$, as a consequence of having polydisperse rather than monodisperse dust, means smaller solitons, other compositional parameters being equal.

\section{Hypergeometric solitary waves}

To ease the numerical discussions about the possibility of having large-amplitude solitary waves with, necessarily, negative polarity, the Sagdeev pseudopotential (23) will be renormalized and written in dimensionless variables, as follows. The electrostatic potential becomes $\psi=e \varphi / T_{\mathrm{i}}$, and velocities are expressed in terms of a reference velocity $V_{\text {ref }}$, defined through $V_{\text {ref }}^{2}=Q T_{\mathrm{i}} / e M a_{\mathrm{min}}^{2}$, so that $u=V / V_{\text {ref }}$ is the dimensionless solitary wave velocity, and sizes are measured in units of $a_{\mathrm{min}}$, with $\alpha=a / a_{\min }$ and $r=a_{\max } / a_{\min }$. Furthermore, $\tau=T_{\mathrm{i}} / T_{\mathrm{e}}$ is the ion-to-electron temperature ratio. Supposing that $r$ is sufficiently large, so that $r \rightarrow \infty$ can be taken with no great harm, for $\beta>4$, the dimensionless charge densities (in absolute value) can be introduced as $f_{\mathrm{e}}=e n_{\mathrm{e} 0} /\left|\sigma_{\mathrm{d} 0}\right|$ and $f_{\mathrm{i}}=e n_{\mathrm{i} 0} /\left|\sigma_{\mathrm{d} 0}\right|$. Hence we obtain

$$
\begin{aligned}
\mathcal{S}(\psi)= & \frac{f_{\mathrm{e}}}{\tau}(1-\exp [\tau \psi])+f_{\mathrm{i}}(1-\exp [-\psi]) \\
& +u^{2}(\beta-2) \int_{1}^{\infty} \alpha^{3-\beta}\left\{1-\sqrt{1+\frac{2 \psi}{\alpha^{2} u^{2}}}\right\} \mathrm{d} \alpha .
\end{aligned}
$$

The dust contribution to $\mathcal{S}(\psi)$ can be integrated in terms of hypergeometric functions ${ }_{2} F_{1}$, but that, in itself, is not very illuminating. What is more revealing is to determine the parameter domains for which nonlinear waves can exist. This will be the emphasis of the discussion in this section.

We recall that the origin is a local maximum for $\mathcal{S}(\psi)$, so that $\mathcal{S}(\psi)<0$ for negative $\psi$ in the immediate vicinity of $\psi=0$, and that, furthermore, $\psi$ is restricted on the negative side to $\psi_{\mathrm{c}}=-u^{2} / 2<\psi<0$. To have solitary wave solutions, $\mathcal{S}(\psi)$ needs to have at least one root $\psi_{r}$ in that interval, and until the first negative root is met, $\mathcal{S}(\psi)$ stays negative. Rephrasing this, for $\psi<\psi_{r}$ but $\psi$ close enough to $\psi_{r}$, we necessarily have that $\mathcal{S}(\psi)>0$. For given compositional parameters, including $f_{\mathrm{i}}$ and $f_{\mathrm{e}}$, the unknown variable is $u$, and as we increase $u$ the solitary wave amplitude becomes larger and $\psi_{r}$ is pushed farther out, towards $\psi_{\mathrm{c}}$. Consequently, the upper limit on $u$ is given by the condition that $\mathcal{S}\left(\psi_{\mathrm{c}}\right)=0$, whereas the lower limit follows from (16), rewritten here as

$u^{2}=\frac{\beta-2}{\beta\left[f_{\mathrm{i}}(1+\tau)-\tau\right]}$,

with the help of the undisturbed charge neutrality (12), in normalized variables

$f_{\mathrm{i}}=f_{\mathrm{e}}+1$.

For $f_{\mathrm{e}}$ to be nonnegative, $f_{\mathrm{i}} \geqslant 1$ is required, a limit that will have to be kept in mind further on. Because we have chosen in our parametric investigation to scale all velocities by a constant $V_{\text {ref }}$, any change in $\beta, \tau$, and $f_{\mathrm{i}}$ will affect the dust-acoustic velocity in the model. Combined with the requirement that the solitary waves be superacoustic, it is then the $u$ given by (33) that represents the true dust-acoustic velocity, in dimensionless notation.

The upper curve following from $\mathcal{S}\left(\psi_{\mathrm{c}}\right)=0$ is given in parametric form by

$$
f_{\mathrm{i}}=\frac{u^{2} C(\beta)+\frac{1}{\tau}\left(1-\exp \left[-\frac{\tau u^{2}}{2}\right]\right)}{1-\exp \left[\frac{u^{2}}{2}\right]+\frac{1}{\tau}\left(1-\exp \left[-\frac{\tau u^{2}}{2}\right]\right)}
$$

where

$C(\beta)=(\beta-2)\left(\frac{\sqrt{\pi} \Gamma\left[\frac{\beta-4}{2}\right]}{4 \Gamma\left[\frac{\beta-1}{2}\right]}-\frac{1}{\beta-4}\right)$.

Here $\Gamma$ stands for the gamma function and the expressions hold for $\beta>4$, because of simplifications like $a_{\min } \ll a_{\max }$. It can be checked that $C(\beta)$ is a well-behaved and continuous function of $\beta$.

For a fixed $f_{\mathrm{i}} \geqslant 1$, any value of $u$ between the lower and upper curves yields negative-polarity solitary waves, the amplitude of which is small for $u$ close to the lower curve and increases as the upper curve is approached. Neither the lower nor the upper curves are included, since the solitary waves are not superacoustic on the lower curve, and on the upper curve the dust density ceases to be real. The advantage of working in an $\left\{f_{\mathrm{i}}, u\right\}$ diagram is that, once the existence regime is known for given compositional parameters, any choice of $f_{\mathrm{i}}$ and $u$ within the admissible region will lead to a correct Sagdeev pseudopotential. Rather than give countless examples of those for which the numerics tell us that it works, it is more enlightening to have the physical limitations of the plasma model clearly set out.

In order to keep the discussion tractable, we use additional simplifications for the ion and electron temperatures. One is that $T_{\mathrm{i}} \sim T_{\mathrm{e}}$, as quite often happens in astrophysical and space plasmas, and we approximate this by taking $\tau=1$. The other extreme is that $T_{\mathrm{i}} \ll T_{\mathrm{e}}$, which corresponds to taking $\tau \rightarrow 0$ and considering the electrons "superhot". The latter assumption results in a linearization of the electron contribution in $\mathcal{S}(\psi)$, and is more typical of laboratory plasmas. Other values of $\tau$ can be considered, of course, but the curves for $\tau=0$ and $\tau=1$ should suffice to illustrate the trend toward increasing $\tau$.

When $\tau=0$, one can analytically prove that the upper and lower curves, given by (33) and (35), cross for values that annul

$$
\frac{\beta \sqrt{\pi} \Gamma\left[\frac{\beta-4}{2}\right]}{\Gamma\left[\frac{\beta-1}{2}\right]}-\frac{2 \beta^{2}}{(\beta-2)(\beta-4)}=\frac{4}{u^{4}}\left(1+\frac{u^{2}}{2}-\exp \left[\frac{u^{2}}{2}\right]\right) \text {. }
$$

The 1.h.s. of (37) is -0.91 for $\beta=4$ and drops monotonically to -2 for $\beta \rightarrow+\infty$, whereas the r.h.s. is also monotonically 


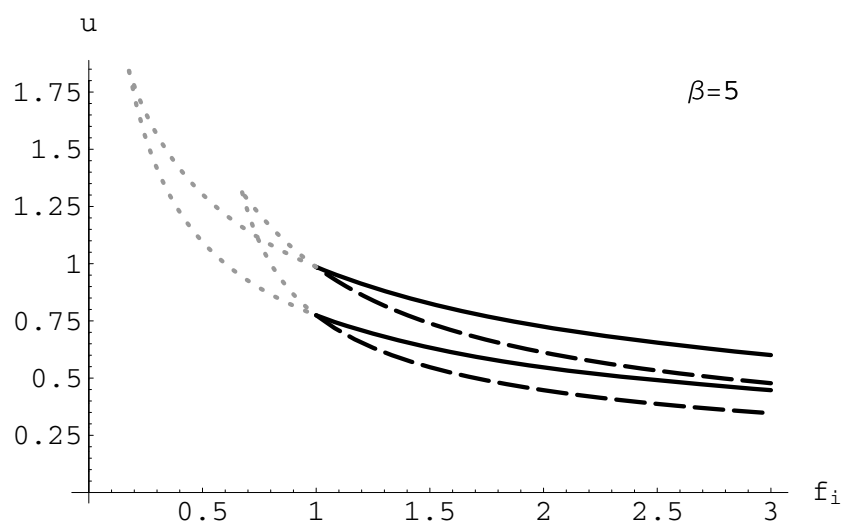

Fig. 1. The lower curves express the minimum (soliton) condition for $u$ at given $f_{\mathrm{i}}$, the upper ones the dust density reality condition, for $\beta=5$ and for $\tau=0$ (full curves) and $\tau=1$ (dashed curves). The gray dotted parts of the curves are not accessible, because $f_{\mathrm{e}}$ would be negative. For a given $f_{\mathrm{i}} \geqslant 1$, admissible $u$ are found between the upper and lower curves.

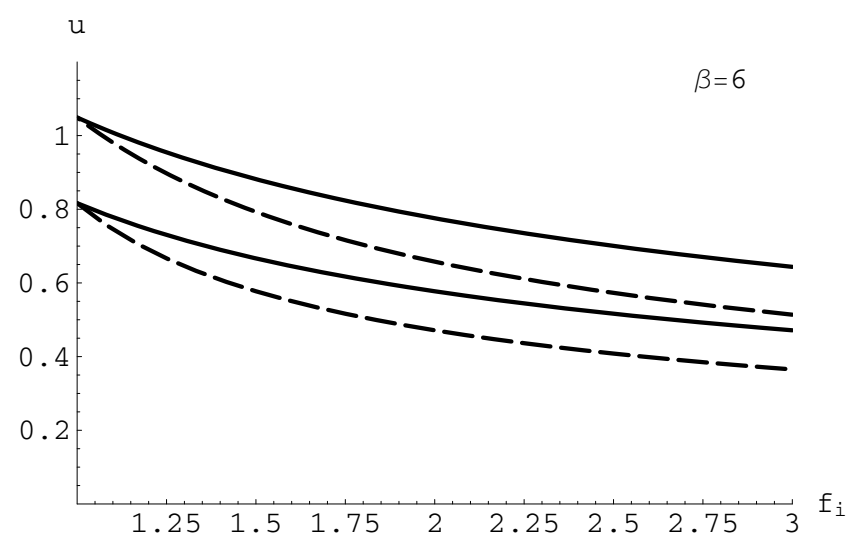

Fig. 2. As Fig. 1, but for $\beta=6$, without the inaccessible parts of the curves.

decreasing from $-1 / 2$ for $u=0$ to $-\infty$ for $u \rightarrow+\infty$. Hence, for any admissible $\beta$, there will be one and only one crossover value for $u$. However, as is illustrated on the existence diagram in Fig. 1 for $\beta=5$, the crossover occurs for too small $f_{i}$. As we have checked numerically, this seems to be the case for a wide range of parameters.

Figures 1-3 indicate the existence ranges in the parameter space $\left\{f_{\mathrm{i}}, u\right\}$, where the admissible $u$ lie between the upper and the lower curves for a given $f_{\mathrm{i}} \geqslant 1$. The lower curves express the minimum (soliton) conditions for $u$ at given $f_{\mathrm{i}}$, the upper ones the dust density reality conditions, for $\tau=0$ as a pair of full curves, and for $\tau=1$ as a pair of dashed curves. For $\beta=5$ we have also continued (in dotted gray) the curves up to the crossover point between lower and upper curves in Fig. 1. These are, however, not accessible because $f_{\mathrm{e}}$ would be negative. A further remark is that the lower curves for different $\tau$ all start from the same point at $f_{\mathrm{i}}=1$, because there $f_{\mathrm{e}}=0$ and the electron temperature is irrelevant. The same holds for the upper curves.

A careful inspection of the different figures reveals that for a given $f_{\mathrm{i}}$ the limits on $u$ increase with $\beta$, both for the upper as for the lower values. The existence domains start at $f_{\mathrm{i}}=1$, where all negative charge is on the dust. This is admittedly an extreme case, but the algebra does not include difficulties up to here. More real situations would have much less negative charge on the dust, hence will lead to much higher $f_{\mathrm{i}}$ values. This is a

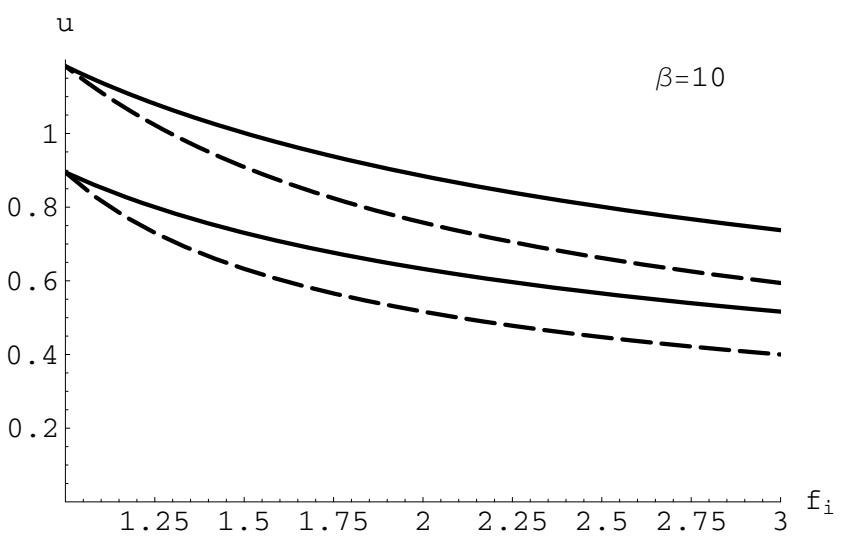

Fig. 3. As Fig. 2, but for $\beta=10$.

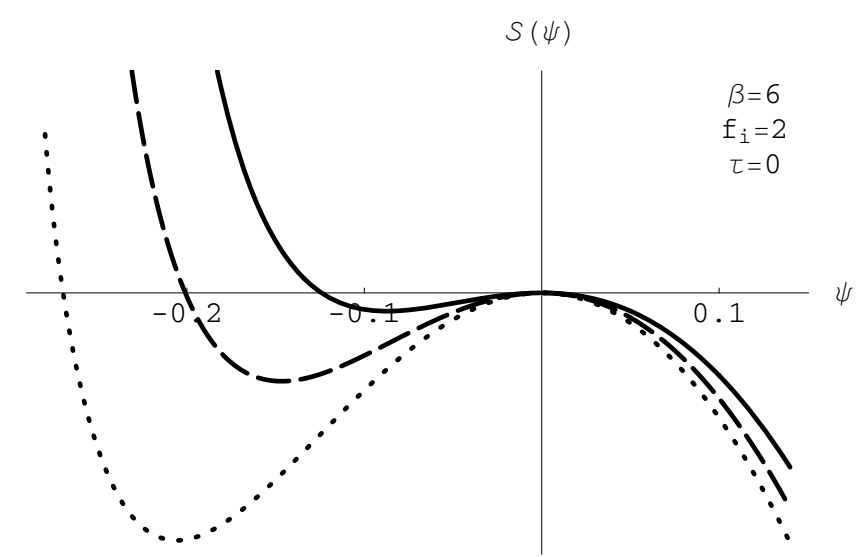

Fig. 4. Generic example of Sagdeev pseudopotentials for $\beta=6, f_{\mathrm{i}}=$ $2, \tau=0$, and $u=0.75$ (dotted curve), $u=0.70$ (dashed curve) and $u=0.65$ (full curve). For negative $\psi$ the pseudopotentials are limited by $\psi_{\mathrm{c}}$, but the Sagdeev pseudopotentials go monotonically down to $-\infty$ for positive $\psi$. For graphical clarity the graphs have only been shown up to $\psi=0.15$.

consequence of the normalization we have chosen, in terms of the dust charge parameters.

In Figs. 1-3 the curves can be continued to the right for larger $f_{\mathrm{i}}$ without obvious limits, but this part has been omitted for reasons of graphical clarity. It is also seen that as $\tau$ increases from 0 to 1 , the admissible $u$ decrease, yielding solitary waves at smaller (relative) amplitude.

We have repeatedly stressed that, for any $f_{\mathrm{i}}$ and $u$ in the existence regions, Sagdeev pseudopotentials are generated that admit negative solitary waves. A generic example of this suffices and is illustrated in Fig. 4, for $\beta=6, f_{i}=2$, and $\tau=0$, for three different $u$. Taking $u=0.75$ (dotted curve) close to the upper limit 0.78 results in a pseudopotential for which $\psi_{r}$ is close to $\psi_{\mathrm{c}}$, where it ceases to exist. For lower values $u=0.70$ (dashed curve) and $u=0.65$ (full curve) the solitary amplitudes decrease, and we have very weak solitary waves close to the lower limit $u=0.58$, but these have been omitted for reasons of graphical clarity. For positive $\psi$ the Sagdeev pseudopotentials are not limited, as discussed in Sects. 3 and 4, and go monotonically down to $-\infty$, but the curves have been limited to $\psi=0.15$.

Increasing $\tau$ gives a similar qualitative picture, shown in Fig. 5, for $\beta=6, f_{\mathrm{i}}=2$, and $\tau=1$, for three different $u$. For $u=0.65$ (dotted curve), $\psi_{r}$ is very close to $\psi_{\mathrm{c}}$, where the graph no longer exists. For lower values $u=0.60$ (dashed curve) and $u=0.55$ (full curve), the solitary amplitudes decrease, and 


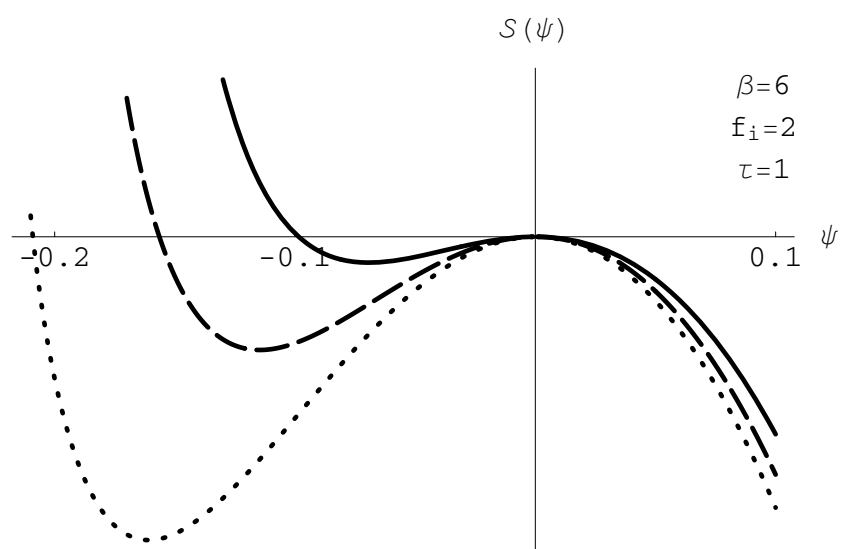

Fig. 5. As Fig. 4, but for $\beta=6, f_{\mathrm{i}}=2, \tau=1$, and $u=0.65$ (dotted curve), $u=0.60$ (dashed curve) and $u=0.55$ (full curve). For graphical clarity the graphs have only been shown up to $\psi=0.1$.

Table 1. Maximal density compression ratios.

\begin{tabular}{c|cccc|c}
\hline \hline$\beta$ & 4 & 6 & 8 & 10 & $\infty$ \\
\hline$v$ & 2.36 & 2.96 & 3.44 & 3.86 & $\infty$ \\
\hline
\end{tabular}

close to the lower limit $u=0.47$ very weak solitary waves occur. The graphs have been limited on the right to $\psi=0.1$. The examples where $\psi_{r}$ is chosen very close to $\psi_{\mathrm{c}}$ yield the largest and fastest solitary waves, as illustrated by the dotted curves in Figs. 4 and 5.

Before closing this section, it is worth speculating about possible observational signatures of solitary structures in dusty plasmas in planetary rings. We expect that localized increases in dust densities should be the first to be seen, eventually, because present-day instrumentation is not geared to that. A closer look at Figs. 4 and 5 indicates that, for nonlinear structures, there is a relation between their velocities and amplitudes, although for larger structures it is not the simple one known from (weakly nonlinear) reductive perturbation analysis, as discussed after (31) in Sect. 4.

For slightly superacoustic structures the amplitudes will be small, and it is therefore interesting to look at the largest compressions possible, before we run into the limitations expressed by (35). Because the minimal grain sizes determine (for $\beta>4$ ) both the actual and the undisturbed dust densities, the density compression ratios are independent of the actual choice for the minimal size. This is illustrated by the expression for the maximum rate $v$,

$v=\left.\frac{n_{\mathrm{d}}}{n_{\mathrm{d} 0}}\right|_{\psi=\psi_{\mathrm{c}}}=\frac{(\beta-1) \sqrt{\pi} \Gamma\left[\frac{\beta-1}{2}\right]}{2 \Gamma\left[\frac{\beta}{2}\right]}$,

obtained for $\psi=\psi_{\mathrm{c}}$ from the integration over size of the dimensionless form of (10). Indicative numbers are given in Table 1. It is interesting to note that for monodisperse dust (corresponding to $\beta \rightarrow \infty$ as discussed in Sect. 6) the compression goes to infinity, as this is one of the physical limitations on the existence domains for that model. This can be seen from (10), when the tildes are omitted and $q$ and $m$ refer to the charge and mass of the (identical) grains, respectively. More remarks about the distinction between the monodisperse case and one with distributed dust are made in Sect. 6.

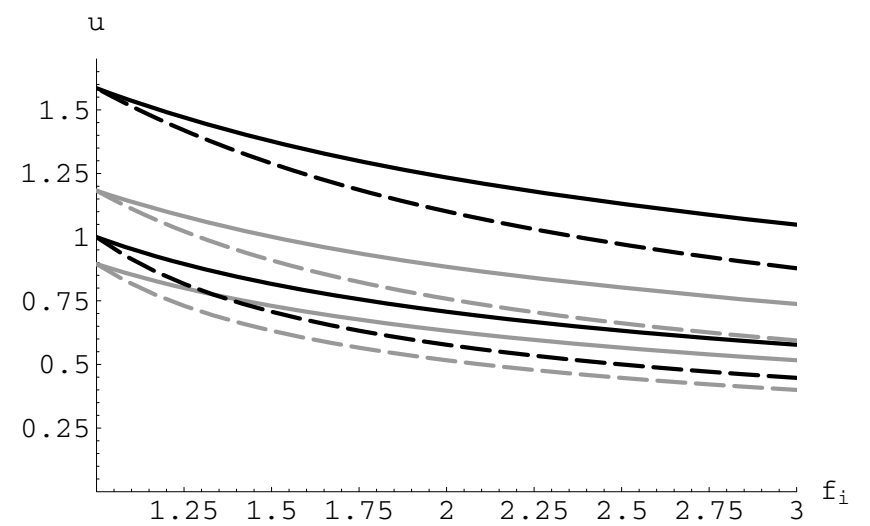

Fig. 6. As Fig. 2, for $\beta=10$ (gray curves) and monodisperse dust (black curves).

\section{Comparison with monodisperse dust}

At this stage, it is interesting to further compare the treatment for different $\beta$ dust distributions to the case of monodisperse dust, with (negative) charge $q_{\mathrm{d}}$ and mass $m_{\mathrm{d}}$, as encountered in many papers (Rao et al. 1990; Bharuthram \& Shukla 1992; Lakshmi \& Bharuthram 1994; Mamun et al. 1996; Ma \& Liu 1997; Singh \& Rao 1997; Mamun 1999; McKenzie 2002; Verheest et al. 2005). For monodisperse dust we have to replace (32) by

$$
\begin{aligned}
\mathcal{S}(\psi)= & \frac{f_{\mathrm{e}}}{\tau}(1-\exp [\tau \psi])+f_{\mathrm{i}}(1-\exp [-\psi]) \\
& +u^{2}\left\{1-\sqrt{1+\frac{2 \psi}{u^{2}}}\right\}
\end{aligned}
$$

in the same normalized variables as used before, except for $V_{\text {ref }}$, which is now defined through $V_{\text {ref }}^{2}=\left|q_{\mathrm{d}}\right| T_{\mathrm{i}} / e m_{\mathrm{d}}$. For superhot electrons, the soliton condition becomes $u^{2}>1 / f_{\mathrm{i}}$, and a comparison with $u^{2}>(\beta-2) / \beta f_{\mathrm{i}}$ indicates that the dust distribution is quasi-monodisperse for very high $\beta$.

The upper limit on $u$ corresponds physically to infinite dust compression at $\psi_{\mathrm{c}}$, and is given by (35), provided $C(\beta)$ is replaced by -1 . As surmised, this corresponds to $\lim _{\beta \rightarrow+\infty} C(\beta)=$ -1 , so that, indeed, large $\beta$ corresponds to effectively monodisperse dust. Again, the upper and lower curves cross once, but in the domain where $f_{\mathrm{i}}<1$ and $f_{\mathrm{e}}$ is no longer real. The results are presented in Fig. 6, for $\tau=0$ (full curves) and $\tau=1$ (dashed curves). We have repeated the curves generated in Fig. 3 in gray, with the same convention. From this comparison it is clear that, at given $f_{i}$, the upper values of $u$ are indeed higher for monodisperse dust than for the finite $\beta$ ranges observed in planetary rings.

As a result, dust size distributions admit slower solitary waves under similar plasma conditions than would be given by monodisperse dust models. This was also noted when discussing weaker nonlinear solutions in Sect. 4. For a reasonable $\beta$, like 6, the difference can be quite appreciable, and a monodisperse treatment would give solitary wave amplitudes which are too large. However, the changes are quantitative rather than qualitative, in the sense that the monodisperse treatment also does not admit positive solutions when the dust is negatively charged.

\section{Conclusions}

A description has been given of large-amplitude, nonlinear solitary waves in space plasmas containing charged dust distributions, based on a Sagdeev pseudopotential treatment that was 
suitably modified compared to earlier work to include mass and charge as additional continuous variables. To deal with planetary dust rings, the grain size was used as a variable, where grain density has a power-law decay with size as commonly observed. First, the Korteweg-de Vries limit was taken, from which it was concluded that only negative potential profiles can exist for negatively charged dust, which is also true for monodisperse dust.

The fully nonlinear case was worked out in terms of existence domains in the space of compositional parameters, with emphasis on the physical reasons for possible restrictions. A lower limit for possible solitary-wave velocities is found by requiring that the Sagdeev pseudopotentials have a local maximum in the undisturbed medium far from the solitary structure. The upper limit follows from reality conditions for the dust distribution, and together, the two limits were illustrated for different $\beta$ as functions of relative densities between the plasma species. Any velocity $u$ lying between the lower and upper curves yields negative-polarity solitary waves, the amplitude of which is small for $u$ close to the lower curve and increases as the upper curve is approached.

Under similar plasma conditions, dust size distributions admit slower solitary waves than given by monodisperse dust models. For values of $\beta$, typical for planetary rings, the difference can be appreciable, and a monodisperse treatment thus gives solitary wave amplitudes that are too large. Increasing the ion temperature with respect to the electron temperature results in a decrease in solitary wave amplitudes. For distributed dust, the maximum density compression at the center of the structure is finite and limited to doubling or tripling, whereas monodisperse models predict unphysically high compressions for the fastest structures.

Finally, since power-law decays are quite generic, the results presented here should also be applicable to other astrophysical plasmas containing charged dust distributions. However, other methods than in situ spacecraft observations will be needed to quantify the dust distributions outside the heliosphere.

Acknowledgements. F.V. thanks the Fonds voor Wetenschappelijk Onderzoek (Vlaanderen) for its support.

\section{References}

Baboolal, S., Bharuthram, R., \& Hellberg, M. A. 1990, J. Plasma Phys., 44, 1 Bale, S. D., Kellogg, P. J., Larson, D. E., et al. 1998, Geophys. Res. Lett., 25, 2929

Bharuthram, R., \& Shukla, P. K. 1992, Planet. Space Sci., 40, 973
Bliokh, P., Sinitsin, V., \& Yaroshenko, V. 1995, Dusty and Self-Gravitational Plasmas in Space (Dordrecht: Kluwer)

Bounds, S. R., Pfaff, R. F., Knowlton, S. F., et al. 1999, J. Geophys. Res., 104, 28709

Brattli, A., Havnes, O., \& Melandsø, F. 1997, J. Plasma Phys., 58, 691

Cattaert, T., Verheest, F., \& Hellberg, M. A. 2005, Phys. Plasmas, 12, 042901

de Juli, M. C., \& Schneider, R. S. 1998, J. Plasma Phys., 60, 243

Franz, J. R., Kintner, P. M., \& Pickett, J. S. 1998, Geophys. Res. Lett., 25, 1277

Gurnett, D. A., Grün, E., Gallagher, D., Kurth, W. S., \& Scarf, F. L. 1983, Icarus, 53,236

Hellberg, M. A., Mace, R. L., \& Cattaert, T. 2005, Space Sci. Rev., 121, 127

Kempf, S., Beckmann, U., Srama, R., et al. 2006, Planet. Space Sci., 54, 999

Kempf, S., Beckmann, U., Moragas-Klostermeyer, G., et al. 2008, Icarus, 193, 420

Kurth, W. S., Averkamp, T. F., Gurnett, D. A., \& Wang, Z. 2006, Planet. Space Sci., 54, 988

Lakshmi, S. V., \& Bharuthram, R. 1994, Planet. Space Sci., 42, 875

Ma, J. X., \& Liu, J. Y. 1997, Phys. Plasmas, 4, 253

Mace, R. L., \& Hellberg, M. A. 1995, Phys. Plasmas, 2, 2098

Mamun, A. A. 1999, Ap\&SS, 268, 443

Mamun, A. A., Cairns, R. A., \& Shukla, P. K. 1996, Phys. Plasmas, 3, 702

Matsumoto, H., Kojima, H., Miyatake, T., et al. 1994, Geophys. Res. Lett., 21, 2915

McKenzie, J. F. 2002, J. Plasma Phys., 67, 353

Meuris, P. 1997, Planet. Space Sci., 45, 1171

Meuris, P., Verheest, F., \& Lakhina, G. S. 1997, Planet. Space Sci., 45, 449

Pickett, J. S., Menietti, J. D., Gurnett, D. A., et al. 2003, Nonlin. Proc. Geophys., 10,3

Raadu, M. A. 2001, IEEE Trans. Plasma Sci., 29, 182

Rao, N. N., Shukla, P. K., \& Yu, M. Y. 1990, Planet. Space Sci., 38, 543

Sagdeev, R. Z. 1966, in Rev. Plasma Phys., 4, ed. M. A. Leontovich, Consultants Bureau, New York, 23

Showalter, M. R., \& Cuzzi, J. N. 1993, Icarus, 103, 124

Showalter, M. R., Pollack, J. B., Ockert, M. E., Doyle, L. R., \& Dalton, J. B. 1992, Icarus, 100, 394

Shukla, P. K., \& Mamun, A. A. 2002, Introduction to Dusty Plasma Physics (Bristol: IOP)

Singh, S. V., \& Rao, N. N. 1997, Phys. Lett. A, 235, 164

Summers, D., \& Thorne, R. M. 1991, Phys. Fluids B, 3, 1835

Tripathi, K. D., \& Sharma, S. K. 1996, Phys. Plasmas, 3, 4380

Varma, R. K. 2000, Phys. Plasmas, 7, 3885

Verheest, F. 2000, Waves in Dusty Space Plasmas (Dordrecht: Kluwer)

Verheest, F., \& Cattaert, T. 2003, Phys. Plasmas, 10, 956

Verheest, F., \& Cattaert, T. 2004a, IEEE Trans. Plasma Sci., 32, 653

Verheest, F., \& Cattaert, T. 2004b, A\&A, 421, 17

Verheest, F., \& Pillay, S. R. 2008, Phys. Plasmas, 15, 013703

Verheest, F., Yaroshenko, V. V., \& Hellberg, M. A. 2002a, Phys. Plasmas, 9, 2479

Verheest, F., Mace, R. L., Pillay, S. R., \& Hellberg, M. A. 2002b, J. Phys. A: Math. Gen., 35, 795

Verheest, F., Hellberg, M. A., \& Yaroshenko, V. V. 2003, Phys. Rev. E, 67, 016406

Verheest, F., Cattaert, T., \& Hellberg, M. A. 2005, Phys. Plasmas, 12, 082308

Verheest, F., Hellberg, M. A., \& Kourakis, I. 2008, Phys. Plasmas, 15, 112309

Yaroshenko, V. V., Jacobs, G., \& Verheest, F. 2001, Phys. Rev. E, 64, 036401

Yaroshenko, V. V., Ratsynskaia, S., Olson, J., et al. 2009, Planet. Space Sci., DOI:10.1016/j.pss.2009.03.002 